REVUE DE L'INSTITUT

FRANÇAIS D'HISTOIRE

EN ALLEMAGNE

\section{Revue de l'IFHA}

Revue de l'Institut français d'histoire en Allemagne

$1 \mid 2009$

IFHA 1

\title{
Le Bildarchiv Foto Marburg, centre allemand de documentation pour l'histoire de l'art
}

\section{Axelle Chassagnette}

\section{OpenEdition}

\section{Journals}

Édition électronique

URL : http://journals.openedition.org/ifha/349

DOI : $10.4000 /$ ifha.349

ISSN : 2198-8943

\section{Éditeur}

IFRA - Institut franco-allemand (sciences historiques et sociales)

\section{Édition imprimée}

Date de publication : 30 septembre 2009

Pagination : 167-170

ISSN : 2190-0078

\section{Référence électronique}

Axelle Chassagnette, «Le Bildarchiv Foto Marburg, centre allemand de documentation pour I'histoire de

l'art », Revue de l'IFHA [En ligne], 1 | 2009, mis en ligne le 07 février 2013, consulté le 30 avril 2019.

URL : http://journals.openedition.org/ifha/349 ; DOl : 10.4000/ifha.349

Ce document a été généré automatiquement le 30 avril 2019.

(CIFHA 


\title{
Le Bildarchiv Foto Marburg, centre allemand de documentation pour l'histoire de l'art
}

\author{
Axelle Chassagnette
}

1 Le Bildarchiv Foto Marburg a le statut de centre national allemand de documentation pour l'histoire de l'art et de l'architecture (Deutsche Dokumentationszentrum für Kunstgeschichte). Il est rattaché à la Philipps-Universität de Marbourg. Fondé en 1913 par l'historien d'art, professeur et photographe expérimenté Richard Hamann (1879-1961), le Bildarchiv trouve son origine dans l'imposante collection de photographies (Photographischer Apparat) rassemblées au sein du département d'histoire de l'art de l'Université. En 1929, cette collection fut désignée par l'État libre de Prusse (Freistaat Preußen) comme centre de documentation sur l'art. En 1962, le centre fut intégré - ou réintégré sous sa nouvelle forme institutionnelle - dans l'Université de Marbourg.

Il a aujourd'hui pour but de collecter, d'inventorier, de numériser et rendre accessibles en ligne des photos conservées sur place ou dans d'autres institutions allemandes (bibliothèques, instituts, musées et monuments) touchant pour l'essentiel à l'histoire de l'art et de l'architecture des pays européens. L'Allemagne constitue le pays le mieux représenté, mais des photos de France, d'Italie, d'Espagne, de Grèce et d'Égypte sont également présentes en nombre dans le fonds. Le Bildarchiv dispose d'une importante équipe à Marbourg ainsi que d'un réseau de correspondants européens qui peuvent contribuer au repérage et au catalogage des documents numérisés, et de partenariats avec une cinquantaine d'institutions. Les medias utilisés pour la consultation sur place ou par internet sont essentiellement la photo, les microfiches ou la photo numérisée, mais les images collectées et numérisées ne sont pas seulement des photographies, quoique celles-ci constituent l'essentiel du fonds : sont aussi représentées d'autres techniques de production et d'autres supports d'images, en particulier des gravures anciennes et modernes, des affiches et des diapositives, qui ont longtemps constitué un outil de prédilection des historiens de l'art. Cela étant, l'accent porté depuis les origines de 
l'institution sur le medium de la photographie a fortement influencé la périodisation du fonds : on n'y trouve guère de reproduction de manuscrits médiévaux - mais les photos contemporaines d'objets et de bâtiments antiques et médiévaux sont nombreuses. Depuis sa fondation, le Bildarchiv n'a cessé d'accroître ses fonds, en particulier par l'acquisition de collections privées. L'institution mène par ailleurs des campagnes régulières de photographie en Allemagne et à l'étranger, en particulier pour documenter des sites ou des monuments dont l'existence est menacée.

Le Bildarchiv possède environ 1,7 million de photos, qui sont consultables sur place et peuvent être reproduites sur demande. Une part importante de ce fonds est consultable en ligne par l'intermédiaire du catalogue développé par le Bildarchiv, le Bildindex für Kunst und Architektur (http://www.bildindex.de/). Ce catalogue en ligne permet de mener une recherche assez fine. Le lecteur dispose pour ce faire de différents critères, qui ne peuvent pas être combinés : artistes, lieu de production, titre, genre, matériau/technique utilisé(e), thèmes, personnes représentées, lieu de découverte, lieu représenté, collection, numéro d'inventaire, datation (première date), datation (seconde date), administrateur de la photo, numéro de prise photographique, photographe. Le catalogue général est également divisé en quatre sous-catalogues dont l'utilisation rend la recherche en ligne plus rapide. Ces catalogues ont été constitués au moyen d'une indexation par noms d'artistes, lieux, portraits et thèmes. L'affichage par ordre alphabétique des titres d'indexation retenus pour chaque catalogue facilite également la recherche. Lorsque l'image recherchée est trouvée, le lecteur peut afficher une notice qui, dans la majorité des cas, comprend une reproduction numérisée de très bonne qualité (l'agrandissement est également possible et permet de visualiser les détails de l'image), une courte description de l'objet matériel, de son format, de son lieu de conservation et parfois de son origine (collection, achat par une institution). La notice fournit également le lien qui permet d'adresser directement au Bildarchiv une commande de reproduction. Le Bildindex propose par ailleurs des sélections thématiques d'images numérisées (sur l'art et l'architecture viennois, les peintres de portraits funéraires en Italie et les portraits issus du Germanisches Nationalmuseum de Nuremberg). La navigation sur le catalogue n'est possible qu'en allemand.

4 Les travaux du Bildarchiv se sont élargis à la recherche sur différents thèmes, traitant de l'utilisation d'images numériques, de l'histoire, de la pratique et de la théorie de la communication des documents visuels. Six chercheurs (dont un professeur) sont actuellement en activité dans l'institution, et participent à différents projets de numérisation, de catalogage ou de réflexion sur l'usage des images. Le Bildarchiv a ainsi participé avec la Staatsbibliothek de Berlin et la Bayerische Staatsbibliothek de Munich au projet des Manuscripta Maediaevalia (voir BullMHFA, 44, 2008, p. 94-97) et travaille actuellement à l'élaboration d'un Kupferstichkabinett (cabinet des estampes) virtuel à partir de la numérisation d'une partie des fonds de gravures (40 000 feuilles en tout) du Herzog Anton Ulrich-Museum de Braunschweig et de la Herzog August Bibliothek de Wolfenbüttel. Il a également développé l'index des portraits gravés à l'époque moderne ( Digitaler Porträtindex ou Porträtindex druckgraphischer Bildnisse der Frühen Neuzeit) qui se trouve maintenant intégré au Bildindex, grâce à un financement de la Deutsche Forschungsgemeinschaft (DFG).

5 Le Bildarchiv de Marburg constitue un partenaire nécessaire pour nombre d'entreprises d'exploitation et de mise en ligne des images comme sources de documentation et de recherche en Allemagne, à tel point qu'il est impossible de mentionner ici tous les projets 
dans lesquels il se trouve engagé. Cette institution constitue une ressource incontournable pour tous les chercheurs et les enseignants intéressés, au-delà de l'histoire de l'art, au domaine de l'iconographie moderne et contemporaine.

\section{ANNEXES}

Bildarchiv Foto Marburg

Biegenstr. 11

D-35 037 Marburg

Allemagne

Tél. (0049) (0)6421 2823600

Fax (0049) (0)6421 2828931

Adresse électronique : bildarchiv@fotomarburg.de

Site internet (en allemand ou en anglais) : www.fotomarburg.de

Catalogue en ligne (en allemand) : http://www.bildindex.de/

\section{AUTEUR}

\section{AXELLE CHASSAGNETTE}

Axelle Chassagnette est bénéficiaire d'une aide à la mobilité à la MHFA / IFHA. 